\section{Caracterización del estado nutricional, hábitos alimentarios y estilos de vida de estudiantes universitarios chilenos: estudio multicéntrico}

\author{
SAMUEL DURÁN ${ }^{1, \mathrm{~b}}$, MIRTA CROVETTO ${ }^{2, \mathrm{a}}$, VALENTINA ESPINOZA ${ }^{3, \mathrm{a}}$, \\ FRANCISCO MENA $^{4, a}$, GLORIA OÑATE ${ }^{5, a}$, MACARENA FERNÁNDEZ ${ }^{5, a}$, \\ SOFÍA COÑUECAR ${ }^{6, \mathrm{a}}$, ÁLVARO GUERRA ${ }^{6, \mathrm{a}}$, MACARENA VALLADARES $^{7, \mathrm{~b}}$
}

\section{Lifestyles, body mass index and sleep patterns among university students}

Background: Health surveys in Chile show a worrisome high prevalence of unhealthy lifestyles among adults. Aim: To characterize the nutritional status, food intake and sleep patterns in university students of both genders. Material and Methods: Cross sectional study in seven Chilean universities. Students from six universities answered a feeding habits survey, the Pittsburgh Sleep Quality Index, Insomnia Severity Index and Epworth Sleepiness Scale. All were weighed and their height was measured. Results: A total of 1,418 students aged $21 \pm 3$ years (22\% males) were evaluated. Three percent were classified as underweight, $68 \%$ as normal weight, $24 \%$ as overweight and $4 \%$ as obese. Thirty three percent of males and $28 \%$ of females smoked. Twenty six percent consumed at least one glass of alcoholic beverages on the weekend, and only $18 \%$ of males and 5\% of females were physically active. Men consumed unhealthy foods with a significantly higher frequency than females. Twenty seven percent had mild daytime somnolence, $24 \%$ had moderate daytime somnolence, 50\% had subclinical insomnia, 19\% moderate insomnia, and $1.4 \%$ had severe insomnia. Conclusions: In this group of students a high frequency of unhealthy lifestyles and malnutrition caused by excess was observed. Also a high prevalence of insomnia, daytime somnolence, and inadequate sleep amounts were recorded.

(Rev Med Chile 2017; 145: 1403-1411)

Key words: Food; Nutritional Status; Sleep; Tobacco.
${ }^{1}$ Facultad de Ciencias de la Salud. Universidad San Sebastián.

Santiago, Chile.

${ }^{2}$ Centro de Estudios Avanzados. Facultad de Ciencias de la Salud. Universidad de Playa Ancha.

Valparaíso.

${ }^{3}$ Carrera de Nutrición y Dietética. Universidad Católica de Temuco. Temuco, Chile.

${ }^{4}$ Departamento de Ciencias de los Alimentos y Nutrición. FACSA. Universidad de Antofagasta. Antofagasta. Chile.

${ }^{5}$ Carrera Nutrición y Dietética,

Facultad de Ciencias de la Salud, Universidad Autónoma de Chile.

Santiago, Chile.

${ }^{6}$ Facultad de Ciencias de la Salud. Universidad de Playa Ancha.

Valparaíso, Chile.

${ }^{7}$ Centro Integrativo de Biología y Química Aplicada (CIBQA).

Universidad Bernardo O'Higgins.

Santiago, Chile.

aNutricionista MSc.

bPhd.

Los autores no declaran conflictos de interés.

Trabajo no recibió financiamiento.

Recibido el 25 de enero de 2017, aceptado el 19 de noviembre de 2017.

Correspondencia a:

Samuel Durán Agüero

Lota 2465, Providencia, Santiago, Chile.

samuel.duran@uss.cl
$\mathrm{E}$ 1 acceso a la educación superior en Chile, ha crecido sostenidamente alcanzando en $\checkmark$ el año 2015, una matrícula de pregrado en institutos profesionales y universidades de 1.152.125 estudiantes ${ }^{1}$, representando más de 5\% de la población total de nuestro país ${ }^{2}$.

La etapa universitaria, se caracteriza por un proceso de transición, de la adolescencia a la edad adulta, que conlleva un aumento de la independencia, la autonomía y la responsabilidad. En esta etapa se toman decisiones autónomas sobre el "cómo, qué, dónde y cuándo comer"3 y es, por lo tanto, crucial para establecer comportamientos y hábitos de salud para toda la vida, incluidos los patrones alimentarios saludables ${ }^{4}$. No obstante, los universitarios, suelen consumir alimentos poco saludables y presentar hábitos de salud inadecuados, afectando su bienestar y aumentar el riesgo de obesidad, diabetes y enfermedad cardíaca coronaria ${ }^{4}$. 
La Encuesta Nacional de Salud (ENS) 2010, mostró que $37,8 \%$ en el rango de edad entre 15 y 24 años, tenían exceso de peso y $29,9 \%$ obesidad abdominal; en el consumo alimentario, menos de $50 \%$ consumían tres o más lácteos, frutas, verduras y cereales integrales al día y, semanalmente pescados o mariscos. Un $45,5 \%$ tenía hábito tabáquico (diario u ocasional), con una edad promedio de inicio los 15,7 años, $14,7 \%$ presentaba un consumo de alcohol de riesgo, perjudicial o dependencia de alcohol con puntaje AUDIT mayor a 8 . Un $20,7 \%$ con actividad física baja y $75,9 \%$ con sedentarismo de tiempo libre (ENS, 2010). Estos datos, sumados a estudios sobre el estado nutricional, consumo de tabaco y hábitos alimentarios en los estudiantes universitarios, confirman que este tema es de gran interés para prevenir el inicio y retardar la aparición de las enfermedades no trasmisibles ${ }^{5,6}$.

Un reciente meta análisis, sobre la ganancia de peso, en los años de permanencia en la universidad, reportó un aumento de $1,5 \mathrm{~kg}$ de peso corporal y $1,1 \%$ en el porcentaje de grasa corporal ${ }^{7}$; explorando en los factores que podrían influir se señalaron, lugar de residencia, actividad física, factores psicosociales, sociodemográficos, y autopercepción de la imagen corporal ${ }^{8,9}$.

El objetivo del presente estudio fue caracterizar y comparar el estado nutricional, hábitos alimentarios y estilos de vida de estudiantes universitarios chilenos, según sexo.

\section{Material y Método}

Estudio transversal, muestra no probabilística. Variables cuantitativas (edad, peso, talla, Índice de Masa Corporal (IMC), circunferencia de cintura, puntaje consumo de desayuno, lácteos, frutas, verduras, pescados, leguminosas, pan, comida casera, bebidas azucaradas, frituras, sal, comida chatarra y galletas, puntaje total comida saludable y no saludable, puntaje Epworth e insomnio, hora de acostarse, latencia del sueño y cantidad de sueño) y cualitativas (estado nutricional, frecuencia de consumo de alimentos, actividad física, consumo de tabaco, de alcohol y patrones de sueño).

La muestra correspondió a 1.418 estudiantes de las universidades: de Antofagasta (Antofagasta), de Playa Ancha (Valparaíso), San Sebastián (Santia- go), Bernardo O’Higgins (Santiago), Autónoma de Chile (Santiago) y Católica de Temuco (Temuco). Se incluyeron todos los estudiantes chilenos de las universidades mencionadas, presentes al momento de la aplicación de la encuesta diseñada para este estudio, (entre los meses de marzo y mayo del año 2016, firmaran el consentimiento informado y respondieran toda la encuesta).

Para la recolección de datos se elaboró una encuesta estructurada, diseñada por los(as) investigadores(as) y aplicada por nutricionistas debidamente capacitadas, con estandarización de los protocolos de aplicación y medición. El estudio fue desarrollado siguiendo la Declaración de Helsinki, respecto al trabajo con seres humanos y presentada al Comité de Ética de la Universidad San Sebastián, quien aprobó la encuesta y su aplicación.

La "Encuesta sobre hábitos alimentarios"10 para medir los hábitos alimentarios y estilo de vida incluyó dos ítems de auto aplicación: 1) Frecuencia de hábitos alimentarios saludables, compuesto por nueve preguntas con respuestas tipo Likert sobre el consumo de desayuno, lácteos, frutas, verduras, pescados, leguminosas y comida casera. Cada pregunta ponderó un puntaje mínimo de 1 (no consume) y máximo de 5 puntos (consume la totalidad de las porciones día/semana sugeridas por las recomendaciones de las guías alimentarias); la sumatoria del puntaje obtenido en cada pregunta, dio como resultado el "Puntaje Total Hábito Alimentario Saludable", con valores entre los 9 a 45 puntos (mayor valor, mejores hábitos alimentarios); 2) Frecuencia de consumo de alimentos o grupo de alimentos no saludables o promotores de ENT: constó de seis preguntas con respuestas tipo Likert sobre bebidas azucaradas, alcohol, alimentos fritos, comida rápida, bocadillos y adición de sal a las comidas, antes de probarlas. Cada pregunta podía ponderar un puntaje mínimo de 1 (no consume) y máximo de 5 (consumo mayor a 3 porciones día/semana), excepto en el caso de la pregunta sobre adición de sal, donde el puntaje mínimo fue de 1 (no consume) y el máximo 3 (consume más de lo recomendado), alcanzando un puntaje total para el ítem, que oscilaba entre 6 y 28 puntos (entre mayor el valor, peores los hábitos alimentarios).

Además, se aplicó el Cuestionario de Insomnio o Insomnia Severity Index (ISI), instrumento de autorreporte, para evaluar la naturaleza, gravedad e impacto del insomnio ${ }^{11,12}$. Contiene 7 preguntas 
que van de 0 a 4 puntos, con puntaje final entre 0 a 28 puntos. Los puntajes totales, clasificaron en función del valor numérico obtenido, de tal forma que los registros logrados se dividen en 4 categorías:

- Ausencia de insomnio clínico (0 a 7 puntos).

- Insomnio subclínico (8 a 14 puntos).

- Insomnio clínico moderado (15 a 21 puntos).

- Insomnio clínico grave (22 a 28 puntos).

Asimismo, se aplicó la escala de somnolencia de Epworth (ESE), que es autoadministrable y evalúa la propensión a quedarse dormido en ocho situaciones sedentarias diferentes, donde cada situación brinda opciones de respuesta, con puntaje que va de 0 a 3 . Al final se obtienen puntajes acumulativos entre 0 y 24 , siendo los puntajes altos los que representan un mayor grado de somnolencia ${ }^{13}$.

Finalmente se preguntó sobre los hábitos de fumar, beber alcohol y realización de actividad física. Se efectuó una medición antropométrica del peso, talla y circunferencia de cintura. La determinación del peso se realizó con un mínimo de ropa, utilizando una balanza mecánica SECA, con capacidad máxima de $220 \mathrm{~kg}$ y una precisión de $50 \mathrm{~g}$ la estatura se midió con el tallímetro incorporado a la balanza y la circunferencia de cintura con una cinta métrica marca SECA, en el punto medio entre la última costilla y la cresta iliaca. El IMC fue calculado dividiendo el peso por la talla al cuadrado y su resultado categorizado, según criterio de la $\mathrm{OMS}^{14}$.

\section{Análisis estadístico}

Se utilizó el programa Microsoft Excel versión 7.0 para la tabulación de los datos y análisis descriptivo, el cual consideró el calculó de la media aritmética y desviación estándar de las variables cuantitativas y de la frecuencia absoluta y relativa de las variables cualitativas. Las variables fueron sometidas a la Prueba de Normalidad de Kolmogorov-Smirnov. Asimismo, la diferencia de media (variables cuantitativas) fue analizada estadísticamente, mediante la prueba t de Student y la diferencia de proporciones (variables cualitativas), a través de la prueba de $\chi^{2}$. Para analizar los factores asociados al sexo se realizó un modelo de regresión logística. Los factores independientes analizados fueron, estado nutricional, actividad física, consumo de tabaco, alimentación y sueño.
Se consideró un nivel de significación de $\alpha<0,05$ con intervalos de confianza de $95 \%$ y el tratamiento estadístico fue realizado con el programa Statistical Package for Social Science (SPSS) versión $22.0^{\circ}$.

\section{Resultados}

Se evaluaron a 1.455 estudiantes, se eliminaron 37 por información incompleta, conformando la muestra de 1.418 universitarios (22,0\% hombres), el promedio de edad, peso, estatura e IMC es de $21,4 \pm 2,7$ años, $62,3 \pm 10,9 \mathrm{k}, 1,62 \pm 0,07 \mathrm{~m}$ y 23,4 $\pm 3,2 \mathrm{k} / \mathrm{m}^{2}$, respectivamente. El $3,4 \%$ calificó con bajo peso, $68,4 \%$ normopeso, $24 \%$ con sobrepeso y $4 \%$ obeso. Según sexo, el 1,5\% y 3,9\% estaban bajo peso, $60 \%$ y $70,7 \%$ normopeso, $32,2 \%$ y $21,7 \%$ con sobrepeso y $6,0 \%$ y $3,5 \%$ con obesidad en los hombres y mujeres, respectivamente (prueba $\chi^{2}, \mathrm{p}<0,001$ ).

En la Tabla 1, se observa que el peso, talla, IMC y circunferencia de cintura es significativamente mayor en los hombres $(\mathrm{p}<0,001)$. Las mujeres presentan mejor puntaje (mayor frecuencia de consumo) en desayuno, frutas, verduras y comida casera y los hombres en pescado, no existiendo diferencias en la suma total de alimentos y preparaciones saludables. En los alimentos o preparaciones poco saludables, los hombres tienen los mayores puntajes en bebidas azucaradas, alcohol, frituras, comida chatarra y las mujeres en galletas, en la suma total de alimentos poco saludables, los hombres tienen significativamente mayor puntaje $(\mathrm{p}<0,05)$.

El 44\% de los estudiantes consume desayuno, $7,2 \%$ consume las porciones recomendadas de lácteos, $7 \%$ frutas y $27 \%$ de verduras. Se observa un elevado consumo de alcohol, comida chatarra y bocadillos dulces. Según sexo, las mujeres presentan mayor frecuencia de consumo semanal/ día en desayuno, lácteos, verduras y menor consumo semanal/día de alcohol y comida chatarra (Figura 1).

El 33,5\% de los hombres y el 28,3\% mujeres consume tabaco $(p=0,043)$, similar a lo que se observa con la actividad física con $17,8 \%$ de los hombres y sólo $5,2 \%$ de las mujeres es activo físicamente $(\mathrm{p}<0,001)$ (Figura 2).

En los patrones de sueño, los hombres se duermen más tarde y presentan una mayor som- 
Tabla 1. Comparación antropométrica, consumo alimentos y patrones de sueño según sexo en universitarios chilenos

\begin{tabular}{|c|c|c|c|}
\hline & $\begin{array}{l}\text { Hombre } \\
n=313\end{array}$ & $\begin{array}{c}\text { Mujer } \\
n=1.105\end{array}$ & Valor $\mathbf{p}$ \\
\hline Edad (años) & $21,5 \pm 3,0$ & $21,4 \pm 2,6$ & 0,815 \\
\hline Peso (k) & $72,8 \pm 11,6$ & $59,3 \pm 8,7$ & 0,001 \\
\hline Estatura (m) & $1,72 \pm 0,06$ & $1,60 \pm 0,05$ & 0,001 \\
\hline $\mathrm{IMC}\left(\mathrm{k} / \mathrm{m}^{2}\right)$ & $24,4 \pm 3,2$ & $23,1 \pm 3,1$ & 0,001 \\
\hline Circunferencia de cintura $(\mathrm{cm})$ & $82,7 \pm 9,3$ & $75,1 \pm 8,3$ & 0,001 \\
\hline Consumo desayuno (puntaje) & $3,6 \pm 1,2$ & $3,9 \pm 1,1$ & 0,001 \\
\hline Lácteos (puntaje) & $2,6 \pm 1,2$ & $2,7 \pm 1,1$ & 0,146 \\
\hline Frutas (puntaje) & $2,8 \pm 1,0$ & $2,9 \pm 1,0$ & 0,084 \\
\hline Verduras (puntaje) & $3,4 \pm 1,1$ & $3,7 \pm 1,1$ & 0,001 \\
\hline Pescados (puntaje) & $2,6 \pm 1,0$ & $2,4 \pm 0,9$ & 0,007 \\
\hline Leguminosas (puntaje) & $2,9 \pm 0,9$ & $2,8 \pm 0,9$ & 0,087 \\
\hline Pan (puntaje) & $2,3 \pm 1,2$ & $2,4 \pm 1,1$ & 0,350 \\
\hline Comida casera (puntaje) & $3,9 \pm 1,2$ & $4,2 \pm 1.1$ & 0,002 \\
\hline Total comida saludable (suma) & $27,1 \pm 5,7$ & $27,6 \pm 5,1$ & 0,159 \\
\hline Bebidas azucaradas (puntaje) & $2,8 \pm 1,3$ & $2,4 \pm 1,1$ & 0,001 \\
\hline Alcohol (puntaje) & $2,4 \pm 1,1$ & $2,0 \pm 1,2$ & 0,001 \\
\hline Frituras (puntaje) & $2,7 \pm 1,2$ & $2,5 \pm 1,0$ & 0,001 \\
\hline Sal (puntaje) & $1,6 \pm 0,7$ & $1,6 \pm 0,7$ & 0,119 \\
\hline Comida chatarra (puntaje) & $2,1 \pm 0,9$ & $2,0 \pm 0,7$ & 0,008 \\
\hline Galletas (puntaje) & $2,2 \pm 1,0$ & $2,4 \pm 0,9$ & 0,010 \\
\hline Total comida no saludable (suma) & $14,1 \pm 4,0$ & $13,0 \pm 3,3$ & 0,001 \\
\hline Epworth (puntaje) & $8,1 \pm 4,8$ & $5,5 \pm 4,6$ & 0,001 \\
\hline Insomnio (puntaje) & $10,2 \pm 5,0$ & $10,4 \pm 4,9$ & 0,479 \\
\hline Hora acostarse & $24: 4 \pm 1: 2$ & $24: 1 \pm 1: 2$ & 0,022 \\
\hline Latencia al sueño (min) & $30,1 \pm 28,3$ & $27,4 \pm 32,3$ & 0,214 \\
\hline Cantidad de sueño (horas) & $8,0 \pm 1,7$ & $7,6 \pm 1,4$ & 0,001 \\
\hline
\end{tabular}

Valores expresados en media \pm DE. Prueba t de Student.

Tabla 2. Comparación porcentual según sexo de somnolencia diurna e insomnio en universitarios

\begin{tabular}{|lccccccc|}
\hline & \multicolumn{2}{c}{ Somnolencia diurna } & \multicolumn{3}{c|}{ Insomnio } \\
& $\begin{array}{c}\text { Normal } \\
(\%)\end{array}$ & $\begin{array}{c}\text { Leve } \\
(\%)\end{array}$ & $\begin{array}{c}\text { Moderada } \\
(\%)\end{array}$ & $\begin{array}{c}\text { Normal } \\
(\%)\end{array}$ & $\begin{array}{c}\text { Insomnio } \\
\text { subclínico }\end{array}$ & $\begin{array}{c}\text { Insomnio } \\
\text { clínico } \\
\text { moderado }\end{array}$ & $\begin{array}{c}\text { Insomnio } \\
\text { clínico } \\
\text { grave }\end{array}$ \\
\hline Mujeres & 60,3 & 30,1 & 9,6 & 29 & 51,3 & 17,4 & 2,2 \\
Hombres & 78,2 & 17,9 & 3,9 & 29 & 49,3 & 20,3 & 1,2 \\
\hline$\chi^{2}$ & & 0,001 & & & & 0,571 & \\
\hline
\end{tabular}




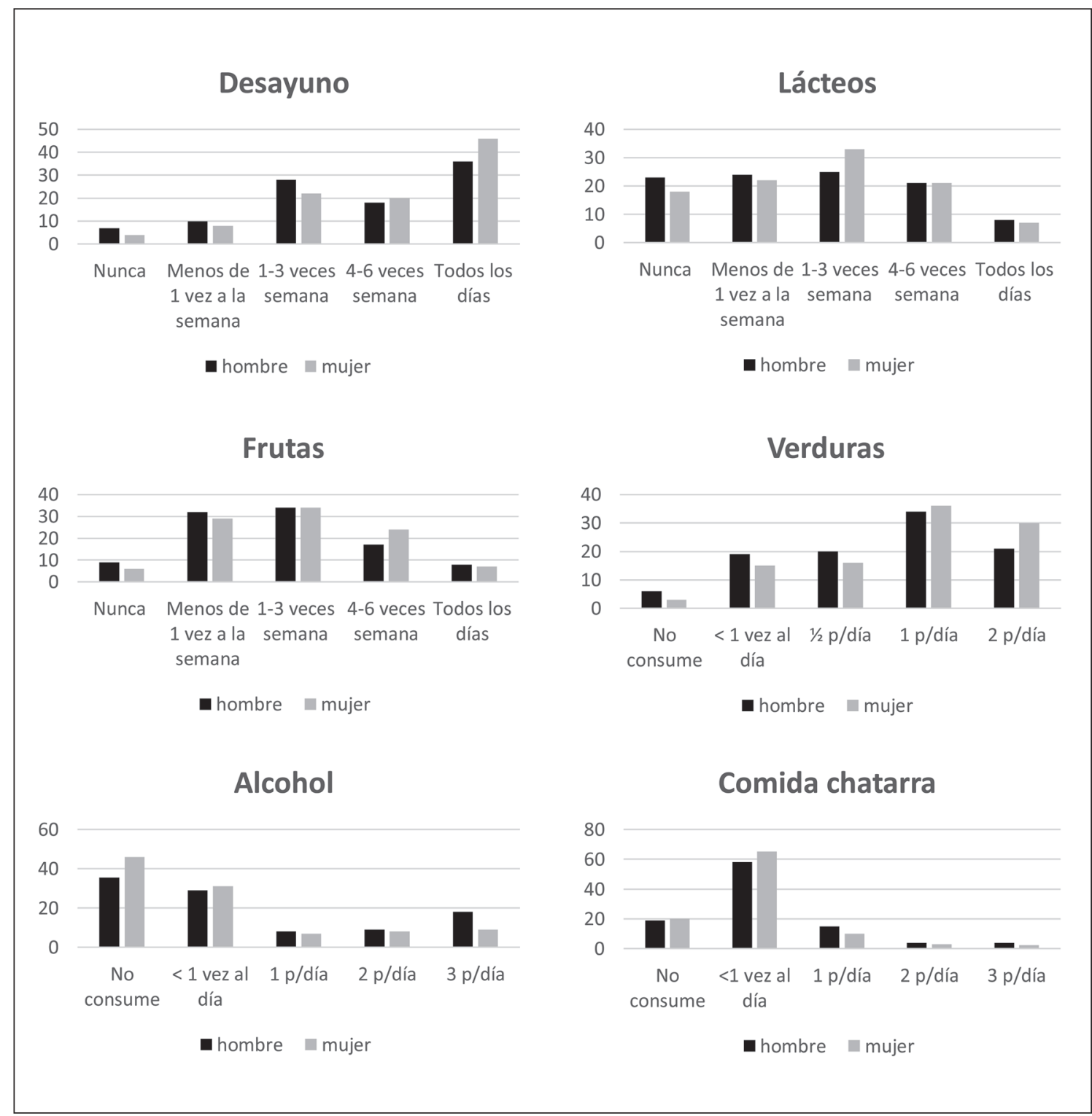

Figura 1. Comparación en la frecuencia de consumo (\%) de algunos alimentos seleccionados según sexo. Prueba $\chi^{2}$ : desayuno $(p<0,001)$; lácteos $(p<0,05)$; frutas $(p=0,064)$; verduras $(p<0,001)$; alcohol $(p<0,01)$; comida chatarra $(p<0,05)$, abreviatura $\mathrm{p}=$ porción.

nolencia diurna y latencia al sueño, las mujeres reportan menos horas de sueño, 64\% no presenta somnolencia diurna, $27 \%$ somnolencia leve y $24 \%$ somnolencia moderada siendo los hombres los que tienen mayor somnolencia diurna $(\mathrm{p}<0,01)$. En insomnio, 29\% no lo presenta y $49,7 \% ; 19 \%$ y $1,4 \%$ de los estudiantes presentan insomnio subclínico, clínico moderado y clínico grave, no presentando diferencias según sexo (Tabla 2).

$\mathrm{Al}$ realizar las asociaciones según sexo, el ser estudiante hombre se asocia a un menor consumo de desayuno, verduras y somnolencia diurna y a mayor consumo de alcohol, alimentos no saludables y mayor latencia al sueño (Tabla 3 ). 


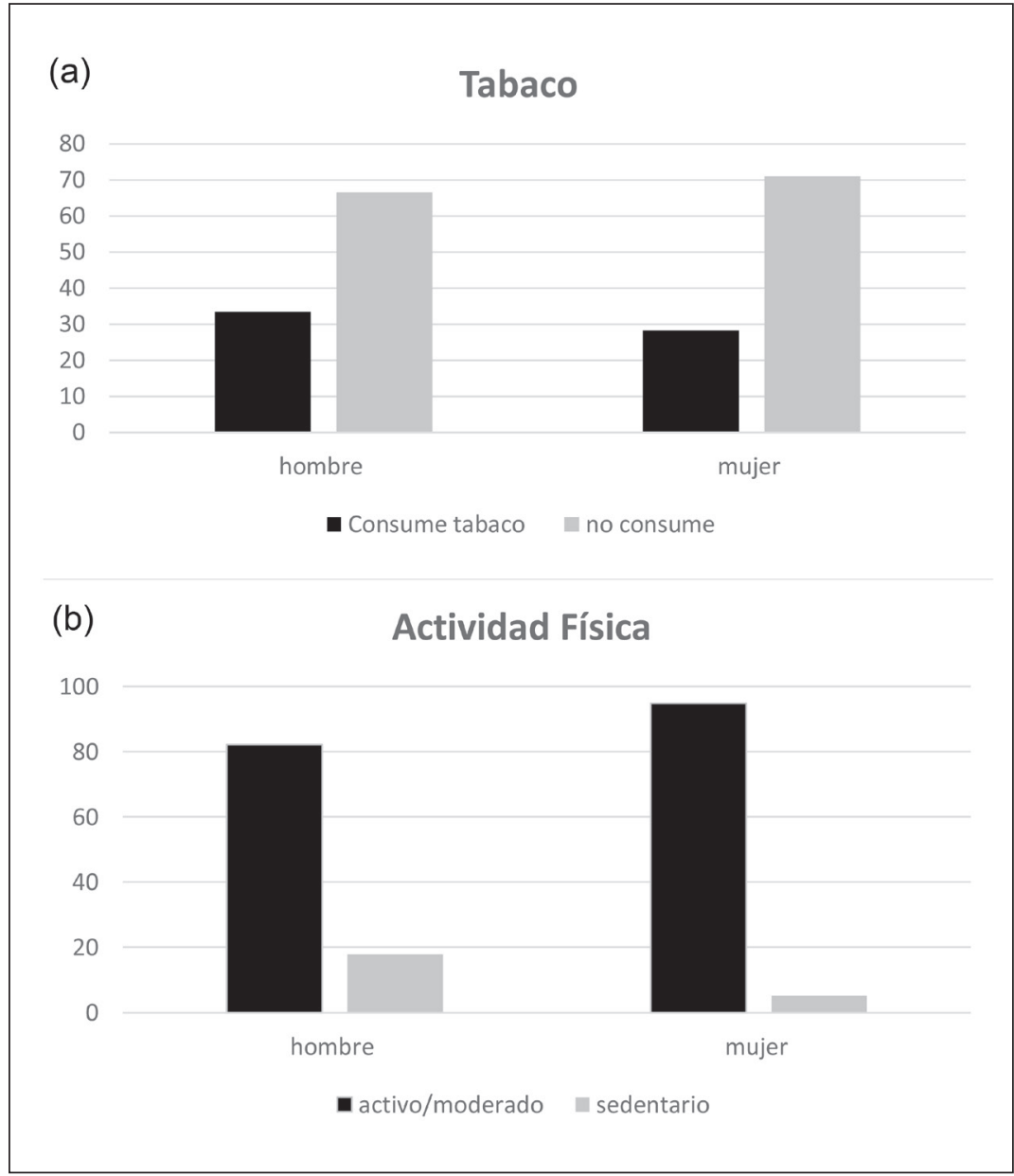

Figura 2. Comparación de consumo de tabaco (a) y actividad física (b) según sexo. Prueba $\chi^{2}$ : Tabaco $p<0,05$; actividad física $p<0,001$.

Tabla 3. Asociación entre el sexo masculino, IMC, frecuencia de consumo de alimentos y sueño

\begin{tabular}{|lccc|}
\hline & OR & IC95\% & Valor $\mathbf{p}$ \\
\hline $\mathrm{IMC}>25$ & 1,688 & $1,160-2,398$ & 0,006 \\
\hline Desayuno infrecuente & 1,420 & $0,982-2,052$ & 0,062 \\
\hline$<2$ porciones/día verduras & 1,573 & $1,021-2,422$ & 0,040 \\
$<3$ porciones/semana de pescado & 0,436 & $0,281-0,679$ & 0,001 \\
\hline$<3$ porciones/semana comida cacera & 1,519 & $1,074-2,148$ & 0,018 \\
\hline Consumo diario de cena saludable & 0,466 & $0,274-0,791$ & 0,005 \\
\hline$>1$ vaso de alcohol & 1,825 & $1,237-2,691$ & 0,002 \\
\hline$>$ latencia al sueño & 1,614 & $1,138-2,288$ & 0,071 \\
\hline$>$ somnolencia diurna & 0,295 & $0,208-0,419$ & 0,001 \\
\hline
\end{tabular}

Ajustado por consumo de tabaco, actividad física, cantidad de sueño, consumo de frutas, bebidas azucaradas, leguminosas, comida chatarra. 


\section{Discusión}

El principal resultado es que un tercio de los estudiantes tiene malnutrición por exceso, alto consumo de alimentos poco saludables y de tabaco, elevado sedentarismo, insomnio, somnolencia diurna, y una cantidad de horas de sueño inadecuada. Por sexo, los hombres tienen peores hábitos alimentarios y mayor consumo de tabaco, alcohol y somnolencia diurna y las mujeres un mayor sedentarismo.

El consumo diario de desayuno es bajo $(44,1 \%)$, menor respecto a otros estudios chilenos con la misma encuesta $(85,5 \%$ y $68 \%$ respectivamente), solo $27,7 \%$ y $7,3 \%$ consume las porciones recomendadas de verduras y frutas, inferior a los reportado en otros estudios ( 45 y 21,8\% de verduras y frutas $)^{15}$, siendo superior en $5 \%$ observado en estudiantes de educación física ${ }^{16}$.

Un estudio realizado en estudiantes de Nutrición observó una asociación positiva entre un peso adecuado y el consumo de verduras $(\geq 2$ raciones al día) OR $=0,66$ (IC 95\% 0,44-0,99), granos enteros OR $=0,47$ (IC 95\% 0,22-1,00), bajo consumo de aperitivos fritos y dulces $\mathrm{OR}=0,64$ (IC $95 \% 0,40-1,01)$ y OR $=0,54$ (IC 95\% 0,36-0,82), respectivamente ${ }^{17}$, en cambio, en estudiantes de educación física se describe una asociación entre el consumo de frutas y un buen estado nutricional ( $\geq 2$ porciones/día) OR $=0,49$ (IC 95\% $0,26-0,92)^{15}$. Chile pese a ser el mayor exportador de frutas del cono sur del mundo, la ingesta no alcanza las recomendaciones ${ }^{18}$.

En otros estudios en universitarios, más de $50 \%$ no consume desayuno diario con diferencias entre hombres (19\%) y mujeres (35\%) en la ingesta de una merienda. Hay una mayor tendencia en las mujeres a ver la televisión, Internet, teléfono móvil y dormir; sin embargo, la única diferencia significativa encontrada fue en el uso de Internet $(\mathrm{p}<0,003)$. La actividad física diaria fue más frecuente en varones $(41,6 \%)$ que en mujeres $(16 \%)$ $(\mathrm{p}<0,001)$, las que tienden $(42,9 \%)$ a dormir durante menos tiempo $(<7 \mathrm{~h})$ que los hombres $(34,4 \%)(\mathrm{p}<0,08)^{19}$.

Otro estudio mostró que $32 \%$ de los estudiantes consume una segunda porción de frutas y verduras; $26 \%$ informa ir más de una vez a la semana a un restaurante de comida rápida y $31 \%$ consume dulces y caramelos varias veces al día ${ }^{20}$. Una mayor adherencia a un patrón de dieta saludable se caracteriza por un alto consumo de alimentos de origen vegetal, lo que se asoció positivamente con una mejor composición corporal, mientras que una mayor adherencia a un patrón de dieta no saludable se caracteriza por un mayor consumo de alcohol, lo que se asoció con mayores concentraciones de lípidos sanguíneos ${ }^{21,22}$.

Del total de los estudiantes evaluados solo 93\% es considerado con actividad moderada y sedentario. Un estudio en universitarios sedentarios, indicó que los principales problemas asociados son fatiga severa $(64,2 \%)$, dolor muscular o articular $(46,3 \%)$, problemas gastrointestinales $(43,1 \%)$ y problemas de sueño $(33,3 \%)^{23}$.

En nuestro estudio, 26\% de los estudiantes consumen $\geq 1$ vaso de alcohol durante el fin de semana, el que es inferior a estudiantes de nutrición con un consumo de $70 \%{ }^{24}$. Otro estudio reportó que $31,3 \%$ de los universitarios consume alcohol, el que señaló que en los primeros años de universidad tenían un doble riesgo de consumo $\mathrm{OR}=1,9$ (IC 95\% 1,1 a 3,1) en comparación con los estudiantes en el quinto año ${ }^{25}$.

Conjuntamente, hay estudios que señalan una asociación directa entre el consumo de alcohol y adiposidad abdominal ${ }^{26}$ así como los aumentos del consumo de alcohol durante la época universitaria el que puede de variar considerablemente ${ }^{27}$, lo que podría explicar en parte un patrón de dieta definida por el consumo de alcohol ${ }^{28}$.

A su vez, diversos estudios muestran que el sueño en cantidad y calidad puede ser el mayor problema de salud pública para los adultos jóvenes que sugieren que los programas de intervención dirigidos a la depresión, control del estrés y patrones saludables de sueño deben estar garantizados ${ }^{29}$.

La deuda de sueño, su mala calidad y la irregularidad en la hora de acostarse pueden, en gran medida, explicar la sensación de somnolencia durante el día y un mayor consumo de sustancias en estudiantes con preferencias vespertinas ${ }^{30}$; en nuestro estudio los participantes se acostaban en promedio después de las 24 h, y 35,8\% tenía somnolencia diurna.

Resultados en investigación en estudiantes de nutrición mostró que 57,1\% dormía menos de la cantidad recomendada, respecto a $76,3 \%$ de nuestro estudio. En otro estudio anterior, dormir menos se asoció con exceso de peso u obesidad $\mathrm{OR}=1,84$ (IC 95\% 1,26-2,68) y también a la somnolencia diurna OR = 1,83 (IC 95\% 1,29-2,76) ${ }^{24}$. 
Otro estudio mostró que $27,8 \%$ de los estudiantes tenía mala calidad de sueño, factor de riesgo para el rendimiento académico y relaciones interpersonales ${ }^{31}$.

La latencia al sueño en nuestro estudio llegó a los $30 \mathrm{~min}$, un estudio en estudiantes universitarios noruegos mostró que la soledad social y emocional dificulta conciliar y mantener el sueño ${ }^{32}$.

Entre las fortalezas del estudio está la muestra alcanzada, las encuestas aplicadas validadas, $y$ entre las debilidades por ser un estudio transversal no podemos describir causalidad.

\section{Conclusión}

Se observa que los estudiantes universitarios presentan malos estilos de vida, en especial en hombres con una elevada prevalencia de malnutrición por exceso, consumo elevado de tabaco y alcohol, elevado sedentarismo, bajo consumo de frutas, verduras, lácteos, y leguminosas y alta frecuencia de consumo de alimentos característicos de la dieta occidental, además problemas importantes de sueño con una elevada prevalencia de insomnio, somnolencia diurna, y una cantidad de sueño inadecuada.

Es necesario realizar políticas de promoción de estilos de vida saludable en las universidades chilenas; mejorías en los comedores; consejería y tratamiento en alimentación, alcohol, tabaco e higiene de sueño y favorecer la actividad física.

\section{Referencias}

1. Consejo Nacional de Educación (CNED) Departamento de Investigación e Información Pública. http://www. cned.cl/public/secciones/SeccionIndicesPostulantes/ OtrasEstadisticas/Tendencias_INDICES_2015.pdf.

2. Evolución Matrícula Educación Superior de Chile Período 1990-2009. http://portales.mineduc.cl/usuarios/1234/ File/Publicaciones/Estudios/5Estudio-Evolucion-Matricula-Historica-1990-2009.pdf.

3. VanKim NA, Larson N, Laska MN. Emerging adulthood: a critical age for preventing excess weight gain? Adolesc Med State Art Rev 2012; 23 (3): 571-88.

4. Nelson MC, Story M, Larson NI, Neumark-Sztainer D, Lytle LA. Emerging adulthood and college-aged youth: an overlooked age for weight-related behavior change.
Obesity 2008; 16 (10): 2205-11.

5. Racette SB, Deusinger SS, Strube MJ, Highstein GR, Deusinger RH. Weight changes, exercise, and dietary patterns during freshman and sophomore years of college. J Am Coll Health 2005; 53 (6): 245-51.

6. Crovetto M, Figueroa B, González L, Jeria A, Ramírez N. Guías alimentarias y su cumplimiento en estudiantes universitarias, Valparaíso, 2013, Chile. Rev Chil Nutr 2015; 42 (2): 164-72.

7. Fedewa MV, Das BM, Evans EM, Dishman RK. Change in weight and adiposity in college students: a systematic review and meta-analysis. Am J Prev Med 2014; 47 (5): 641-52.

8. Finlayson G, Cecil J, Higgs S, Hill A, Hetherington M. Susceptibility to weight gain. Eating behaviour traits and physical activity as predictors of weight gain during the first year of university. Appetite 2012; 58 (3): 1091-8.

9. Provencher V, Polivy J, Wintre MG, Pratt MW, Pancer SM, Birnie-Lefcovitch S, et al. Who gains or who loses weight? Psychosocial factors among first-year university students. Physiol Behav 2009; 96 (1): 135-41.

10. Durán S, Valdés Badilla P, Godoy A, Herrera T. Hábitos alimentarios y condición física en estudiantes de pedagogía en educación física. Rev Chil Nutr 2014; 41 (3): 251-9.

11. Bastien $\mathrm{CH}$, Vallieres A, Morin CM. Validation of the Insomnia Severity Index as an outcome measure for insomnia research. Sleep Medicine 2001; 2 (4): 297-307.

12. Morin MC. Insomnia: Psychological assessment and management. New York: Guilford Press; 1993.

13. The Epworth Sleepiness Scale (ESS). http://epworthsleepinessscale.com/about-the-ess/.

14. World Health Organization. Obesity: preventing and managing the global epidemic. Report of a WHO Consultation. Geneva: World Health Organization; 2000.

15. Musaiger AO, Al-Khalifa F, Al-Mannai M. Obesity, unhealthy dietary habits and sedentary behaviors among university students in Sudan: growing risks for chronic diseases in a poor country. Environ Health Prev Med 2016; 21 (4): 224-30.

16. Godoy Cumillaf A, Valdés Badilla P, Fariña Herrera C, Cárcamo Mora F, Medina Herrera B, Meneses Sandoval E, et al. Association between Fitness, Nutritional Status and Academic Performance in Physical Education Students. Nutr Hosp 2015; 32 (4): 1722-8.

17. Durán Aguero S, Fernández Godoy E, Fuentes Fuentes J, Hidalgo Fernández A, Quintana Muñoz C, Yunge Hidalgo W, et al. Food Patterns Associated with a Healty Body Weight in Chilean Students of Nutrition and Dietetics. Nutr Hosp 2015; 32 (4): 1780-5.

18. Encuesta Nacional de Consumo Alimentario. Informe 
Final. Chile. http://web.minsal.cl/sites/default/files/ ENCA-INFORME_FINAL.pdf.

19. Musaiger AO, Awadhalla MS, Al-Mannai M, AlSawad M, Asokan GV. Dietary habits and sedentary behaviors among health science university students in Bahrain. Int J Adolesc Med Health. 2015 [Epub ahead of print].

20. Hadjimbei E, Botsaris G, Gekas V, Panayiotou AG. Adherence to the Mediterranean Diet and Lifestyle Characteristics of University Students in Cyprus: A Cross-Sectional Survey. J Nutr Metab 2016; 2016: 2742841.

21. Blondin SA, Mueller MP, Bakun PJ, Choumenkovitch SF, Tucker KL, Economos CD. Cross-Sectional Associations between Empirically-Derived Dietary Patterns and Indicators of Disease Risk among University Students. Nutrients 2015; 8 (1). pii: E3.

22. Al-Qahtani MH. Dietary Habits of Saudi Medical Students at University of Dammam. Int J Health Sci (Qassim) 2016; 10 (3): 353-62.

23. Ahn SH, Um YJ, Kim YJ, Kim HJ, Oh SW, Lee CM, et al. Association between Physical Activity Levels and Physical Symptoms or Illness among University Students in Korea. Korean J Fam Med 2016; 37 (5): 279-86.

24. Durán-Aguero S, Fernández-Godoy E, Fehrmann-Rosas P, Delgado-Sánchez C, Quintana-Muñoz C, Yunge-Hidalgo W, et al. Fewer hours of sleep associated with increased body weight in chilean university nutrition students. Rev Peru Med Exp Salud Pública 2016; 33 (2): 264-8.

25. Miquel L, Rodamilans M, Gimenez R, Cambras T, Canudas AM, Gual A. Alcohol consumption in college students from the pharmacy faculty. Adicciones 2016; 27 (3): 190-7.

26. Sayon-Orea C, Martínez-González MA, Bes-Rastrollo M. Alcohol consumption and body weight: a systematic review. Nutr Rev 2011; 69 (8): 419-31.

27. White A, Hingson R. The burden of alcohol use: excessive alcohol consumption and related consequences among college students. Alcohol Res 2013; 35 (2): 201 18.

28. Lenk KM, Erickson DJ, Nelson TF, Winters KC, Toomey TL. Alcohol policies and practices among four-year colleges in the United States: prevalence and patterns. J Stud Alcohol Drugs 2012; 73 (3): 361-7.

29. Wallace DD, Boynton MH, Lytle LA. Multilevel Analysis Exploring the Links between Stress, Depression, and Sleep Problems among Two-Year College Students. J Am Coll Health 2016: 1-9.

30. Bakotic M, Radosevic-Vidacek B, Koscec Bjelajac A. Morningness-eveningness and daytime functioning in university students: the mediating role of sleep characteristics. J Sleep Res. 2016. [Epub ahead of print].

31. Wang L, Qin P, Zhao Y, Duan S, Zhang Q, Liu Y, et al. Prevalence and risk factors of poor sleep quality among Inner Mongolia Medical University students: A cross-sectional survey. Psychiatry Res 2016; 244: 243-8.

32. Hayley AC, Downey LA, Stough C, Sivertsen B, Knapstad M, Overland S. Social and emotional loneliness and self-reported difficulty initiating and maintaining sleep (DIMS) in a sample of Norwegian university students. Scand J Psychol 2017; 58 (1): 91-9. 